\title{
Few-layer black phosphorus and boron-doped graphene based heteroelectrocatalyst for enhanced hydrogen evolution
}

Received 00th January 20xx, Accepted 00th January 20xx

DOI: $10.1039 / \times 0 \times x 00000 x$

\author{
Selengesuren Suragtkhuu, ${ }^{a}$ Munkhjargal Bat-Erdene, ${ }^{b}$ Abdulaziz S. R. Bati, ${ }^{b}$ Joseph G. Shapter, ${ }^{b}$ \\ Sarangerel Davaasambuu*a and Munkhbayar Batmunkh ${ }^{*}$,c
}

\section{Introduction}

The hydrogen evolution reaction (HER) is an economically profitable and eco-friendly way to produce clean hydrogen energy via electrochemical water splitting. Of particular importance in the HER process is to promote the reduction of hydrogen with a minimum overpotential and reduce the additional energy consumption using efficient catalysts. ${ }^{1}$ The best-known HER catalysts to date are platinum (Pt) and its derivatives, which require very low overpotentials, while showing small Tafel slopes and high exchange current densities due to their minimum binding energies with hydrogen. ${ }^{2}, 3$ However, the scarcity and high cost of these Pt based catalysts limit their widespread application. Therefore, the search for efficient and low-cost catalysts that are readily available for use as an alternative to the expensive $\mathrm{Pt}$ catalyst has been the subject of intense investigation over the past several years. Atomically thin two-dimensional (2D) materials have recently attracted much attention for energy related applications. ${ }^{4-8}$ In particular, due to its unique structure and excellent properties such as anisotropic transport, high carrier mobility and tunable bandgap, few-layer black phosphorus (FL-BP) - a layered 2D semiconductor - has shown great promise for use in many electrocatalysis reactions including nitrogen reduction reaction (NRR), ${ }^{9}$ oxygen evolution reaction, ${ }^{10}$ oxygen reduction reaction ${ }^{11}$ and HER. ${ }^{12}$ FL-BP has emerged as one of the most promising candidates among 2D family materials for many applications. ${ }^{13,} 14$ Pumera and his colleagues studied the catalytic properties of electrochemically exfoliated BP for the HER. ${ }^{15}$ Although some enhancement in the HER performance was observed as compared to the bulk BP, the exfoliated BP still exhibited poor electrocatalytic activity. Several recent computational studies suggested based on density functional theory (DFT) calculations that a pure phosphorene nanosheet is catalytically inert for HER due either to the poor conductivity or limited active sites, so suitable modifications such as introducing functionalization and atomic defects are of great importance. ${ }^{16,17}$ Shao et al. ${ }^{18}$ experimentally showed that $\mathrm{NH}_{2}$ functionalized FL-BP nanosheets can exhibit enhanced electrocatalytic HER performance with an overpotential of 290 $\mathrm{mV}$ at $10 \mathrm{~mA} \mathrm{~cm}^{-2}$ in an alkaline electrolyte. Activating FL-BP nanosheets using metal compounds such as Pt and PtRu has been found to be an effective strategy to achieve superior HER performance (better than the traditional Pt/C catalyst). ${ }^{19,} 20$ Despite their outstanding performance, these efficient catalyst materials still use high-cost noble metals.

One promising strategy is to build heterostructures using FL-BP nanosheets with other catalytic materials such as $\mathrm{MoS}_{2}$, MXene $\left(\mathrm{Ti}_{3} \mathrm{C}_{2} \mathrm{~T}_{\mathrm{x}}\right)$ and graphene derivatives. ${ }^{12,} 21,22$ In 2017, He et al. ${ }^{12}$ deposited catalytically active $\mathrm{MoS}_{2}$ flakes onto BP nanosheets to construct a $\mathrm{MoS}_{2}-\mathrm{BP}$ heterostructure. The as-prepared $\mathrm{MoS}_{2}-$ $\mathrm{BP}$ nanosheets showed remarkable HER performance. Due to the electron accumulation on $\mathrm{MoS}_{2}$, the intrinsic exchange current density (jo) of $\mathrm{MoS}_{2}-\mathrm{BP}$ reached $0.66 \mathrm{~mA} \mathrm{~cm}{ }^{-2}$, which was 22 times higher than that of a bare $\mathrm{MoS}_{2}$.

\footnotetext{
a. Department of Chemistry, School of Arts and Sciences, National University of Mongolia, Ulaanbaatar 14200, Mongolia.E-mail: sarangerel@num.edu.mn

b. Australian Institute for Bioengineering and Nanotechnology, The University of

Queensland, Brisbane, Queensland 4072, Australia. E-mail: m.batmunkh@uq.edu.au

c. Centre for Clean Environment and Energy, Griffith University, Gold Coast, Queensland 4222, Australia. E-mail: m.batmunkh@griffith.edu.au

Electronic Supplementary Information (ESI) available: [details of any supplementary information available should be included here]. See DOI: 10.1039/x0xx00000x
} 


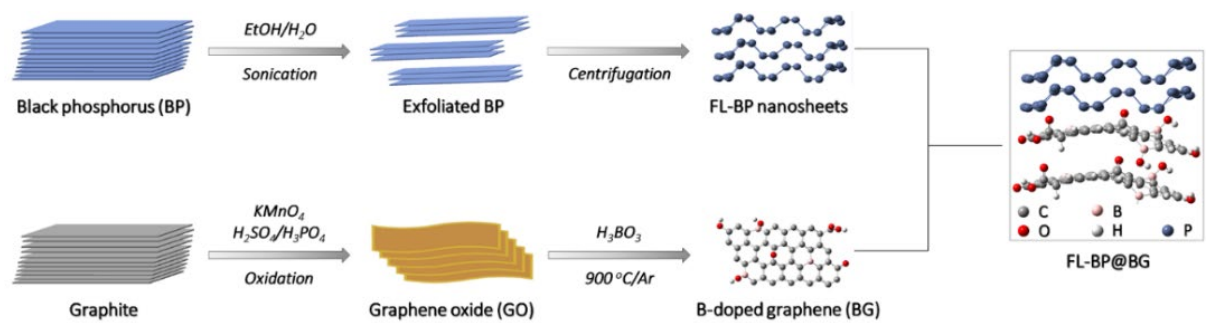

Fig. 1 Preparation of FL-BP nanosheets, BG and FL-BP@BG heterostructure.

Recently, Dai and his colleagues coupled ultrathin FL-BP nanosheets with $\mathrm{N}$-doped graphene (NG) to prepare a metalfree $2 D / 2 D$ heterostructure. ${ }^{22}$ The electronic interaction in this heterostructure induced directional interfacial electron transfer; and thus enriched the electron density over FL-BP and optimized $\mathrm{H}$ adsorption/desorption to promote the HER due to the lower Fermi level of FL-BP as compared to NG. Impressive enhancement in the electrocatalytic HER performance was observed using this $2 \mathrm{D} / 2 \mathrm{D}$ heterostructure relative to the bare FL-BP and NG. Despite these great advancements, the search for novel electrocatalysts is still an active area of research. Herein, we report recent results investigating the catalytic properties of newly designed metal-free heteroelectrocatalysts based on FL-BP nanosheets prepared via liquid phase exfoliation and boron-doped graphene (BG) for the HER in acidic media. It should be noted that our BG was prepared from naturally abundant graphite flakes (obtained from a Mongolian mine $)^{23}$. By coupling FL-BP nanosheets with BG, greatly enhanced HER activity was achieved using FL-BP@BG as compared to the bare FL-BP and BG. In addition to the enhanced catalytic activity, the heteroelectrocatalyst (FL-BP@BG) exhibited excellent stability over 500 continuous cycles.

\section{Results and discussion}

In this work, we first prepared FL-BP nanosheets and BG, followed by hybridizing them using a solution-processed method to obtain a FL-BP@BG heterostructure. The preparation procedure of our BG supported FL-BP (FL-BP@BG) hybrid is shown in Fig. 1. FL-BP nanosheets were prepared using a liquid exfoliation of bulk BP in a mixture of ethanol and water $(4: 1 \mathrm{v} / \mathrm{v})$. It is well known that FL-BP nanosheets tend to rapidly oxidize, which is considered to be a key challenge for many applications. ${ }^{24,25}$ Recently, Xu et al. ${ }^{9}$ found that the oxidation of phosphorene increases the surface hydrophobicity and thus hinders the HER process. To try and limit the oxidation and/or degradation of the FL-BP, the solvent mixture (ethanol and water) was degassed using argon (Ar) gas before being used. Then, bulk BP crystals were added into the solvent mixture, followed by sonication assisted exfoliation. The stable dispersion of FL-BP sheets was obtained by centrifuging the sonicated solution (see Fig. 1 and Fig. S1).

Fig. 2a depicts the Raman spectrum of our FL-BP nanosheets on a silicon ( $\mathrm{Si}$ ) substrate. Notable Raman modes centered at 361 , 437 , and $466 \mathrm{~cm}^{-1}$, which can be assigned to the $A_{g}{ }^{1}, B_{2 g}$, and $A_{g}{ }^{2}$ phonon modes, respectively, were observed and are consistent with previous studies. ${ }^{26,} 27$ We further characterized our FL-BP nanosheets using an X-ray photoelectron spectroscopy (XPS). As shown in Fig. 2b, the high-resolution $P 2 p$ spectrum shows two sharp peaks at $130.0 \mathrm{eV}$ and $130.9 \mathrm{eV}$ due to the $P 2 \mathrm{P}_{3 / 2}$ and $P$ $2 \mathrm{P}_{1 / 2}$ doublet, respectively. ${ }^{28}$ However, our FL-BP sample also showed a peak in the high-energy region at around $134.6 \mathrm{eV}$, which can be assigned to the characteristic peak for oxidized phosphorene $\left(\mathrm{PO}_{\mathrm{x}}\right)$. Interestingly, it should be noted that the intensity of this peak at $134.6 \mathrm{eV}\left(\mathrm{PO}_{\mathrm{x}}\right)$ was low, indicating that our FL-BP nanosheets were only slightly oxidized as a result of degasification of the solvent mixture before exfoliation. For comparison, we also prepared FL-BP nanosheets in the mixed solvent (ethanol and water) without degassing using Ar gas. Fig. S2 shows the high-resolution P $2 p$ spectrum of oxidized FL-BP nanosheets, suggesting that degassing solvent is an important step to protect the FL-BP from oxidation. Moreover, UV-vis spectrum of our FL-BP dispersion, displayed in Fig. 2c, is in excellent agreement with reported literature. ${ }^{29-31}$
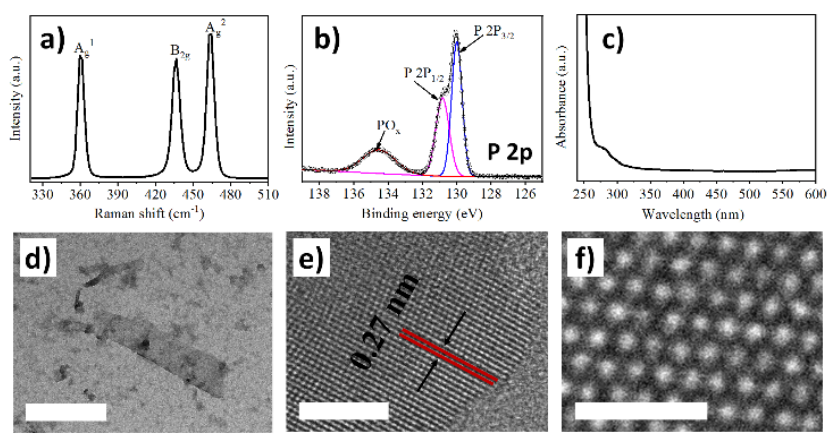

Fig. 2 (a) Raman spectrum, (b) high-resolution XPS P 2p spectrum, (c) UV-vis spectrum, (d) TEM image (scale bar: $400 \mathrm{~nm}$ ), and (e and f) HRTEM image (scale bar: e) $5 \mathrm{~nm}$ and f) $1 \mathrm{~nm}$ ) of FL-BP nanosheets.
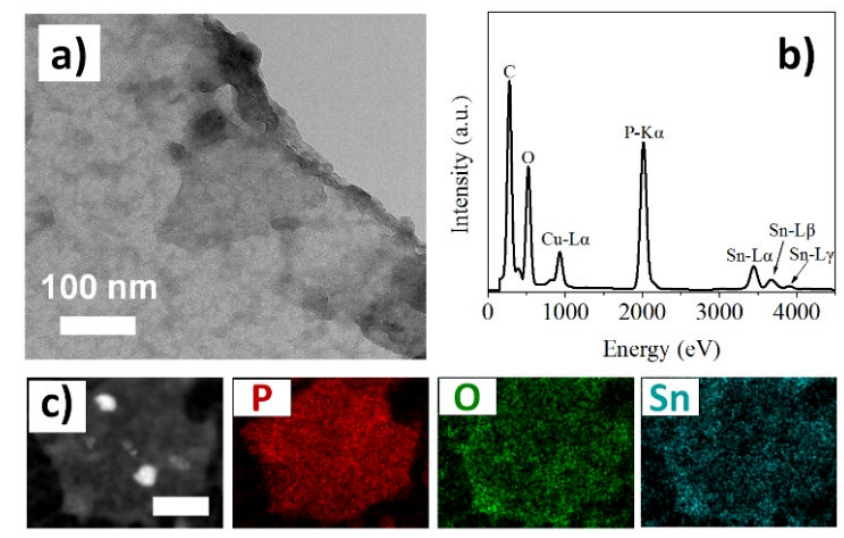

Fig. 3 (a) TEM image and (b) the corresponding EDX spectrum of the FL-BP sheets. (c) HAADF-STEM image (scale bar: $100 \mathrm{~nm}$ ) and the corresponding elemental mapping images for $P, O$, and $S n$ elements. 
Fig. $2 d$ displays the transmission electron microscopy (TEM) image of our FL-BP with a lateral size of around $500 \mathrm{~nm}$. It can also be observed from the TEM image that our nanosheet is transparent, suggesting that ultrathin FL-BP flakes have been obtained after exfoliation. High-resolution TEM (HRTEM) images illustrated in Fig. $2 \mathrm{e}$ and $2 \mathrm{f}$ suggest that our FL-BP nanosheets are highly crystalline, and their lattice spacing was measured to be $\sim 0.27 \mathrm{~nm}$, which is in excellent agreement with previously reported values. ${ }^{32}$ Representative HRTEM images of our FL-BP nanosheet are also illustrated in Fig. S3. The average thickness and flake size of the FL-BP nanosheets were also measured from the atomic force microscopy (AFM). Fig. S4 shows the representative AFM image of few-layer FL-BP nanosheets. The average thickness of the nanosheets was 7.5 $\mu \mathrm{m}$, while the measured flake size was around $500 \mathrm{~nm}$ which is consistent with the TEM measurement.

We further studied the chemical composition of our FL-BP nanosheets using a TEM (Fig. 3a), energy-dispersive X-ray spectroscopy (EDX) (Fig. 3b), and high-angle annular dark-fieldscanning transmission electron microscope (HAADF-STEM) combined with elemental mapping images (Fig. 3c). EDX spectrum depicted in Fig. $3 \mathrm{~b}$ was collected by scanning on the TEM image (Fig. 3a). For the FL-BP, Cu and Sn elements were detected besides $\mathrm{P}$ and $\mathrm{O}$ elements. The appearance of $\mathrm{Cu}$ was expected due to the use of TEM grid, while $S n$ is commonly used for the synthesis of BP crystals. As shown in Fig. 3c and Fig. S3c, the elemental mapping images suggest that our FL-BP sample consists of mainly $P$ and $O$ with a small amount of $\mathrm{Sn}$. These are in excellent agreement with our EDX and XPS results.

Meanwhile, we prepared BG using thermal annealing of graphene oxide (GO) in the presence of boric acid. ${ }^{33}$ It should be noted that the GO was prepared from naturally abundant graphite flakes according to our recent protocol. ${ }^{23}$ For comparison, we also prepared reduced graphene oxide ( $\mathrm{rGO}$ ) flakes by thermal annealing of GO in the absence of boric acid. Raman spectra of the $\mathrm{rGO}$ and $\mathrm{BG}$ deposited on Si substrates are displayed in Fig. 4a. Clearly, both rGO and BG samples show characteristic $D$ and $G$ bands located at $1345 \mathrm{~cm}^{-1}$ and $1577 \mathrm{~cm}$ 1 , respectively. The $D$ and $G$ bands are the predominant features in the spectrum of carbon materials, including graphene derivatives. ${ }^{34}$ The $D$ band involves one phonon, and one defect induced by the second-order double-resonance process and are ascribed to the disorder peak. ${ }^{35}$ On the other hand, the $\mathrm{G}$ band (graphitic peak) corresponds to the in-plane vibrational $E_{2 g}$ mode of the $\mathrm{sp}^{2}$ carbon. ${ }^{36}$ Importantly, the intensity ratio of the $D$ and $G$ bands $\left(I_{D} / I_{G}\right)$ is used to evaluate the structural defect of carbon materials. ${ }^{36}$ Therefore, the $\mathrm{I}_{\mathrm{D}} / \mathrm{I}_{\mathrm{G}}$ ratios were calculated to be 1.03 and 1.44 for the $\mathrm{rGO}$ and $\mathrm{BG}$, respectively. The increase in the $D$ band intensity of $B G$ can be explained by an increase in the structural defects and disorder in the $\mathrm{sp}^{2}$ carbon caused by the introduction of boron atoms. ${ }^{37}$ Such phenomena were observed in earlier studies. ${ }^{38}$ Furthermore, the structural morphology of our BG was investigated using a SEM (see Fig. $4 \mathrm{~b})$, which exhibits the multilayer graphene sheets crumpled together.

XPS is a powerful tool to verify the successful doping and functionalization of nanostructured materials. Fig. 4c shows the XPS survey scan of rGO and BG. Three main peaks, namely carbon (C), oxygen (O), and boron (B), were observed for the BG sample, indicating the successful B-doping on the rGO sheets, while no boron peak was detected for the rGO sample. Fig. $4 \mathrm{~d}$ $f$ displays the high-resolution B 1s, C 1s, and O 1s XPS spectra of the BG. As shown in Fig. 4d, three significant peaks at the binding energies of $190.6 \mathrm{eV}, 192.5 \mathrm{eV}$, and $195.7 \mathrm{eV}$, which can be assigned to the $\mathrm{BC}_{3}, \mathrm{BC}_{2} \mathrm{O}$, and $\mathrm{BCO}$, respectively, were observed. ${ }^{39}, 40$ In particular, the predominant peak observed at $\sim 192.5 \mathrm{eV}$ presents the chemical bonding of boron with carbon and oxygen, ${ }^{38}$ suggesting the successful B-doping of $\mathrm{rGO}$ sheets. As depicted in Fig. 4e, the high-resolution XPS C 1s spectrum shows a high-intensity peak at $284.3 \mathrm{eV}$ due to the $\mathrm{C}=\mathrm{C}$ binding. Additionally, a few other low-intensity peaks, including C-O, $\mathrm{C}=\mathrm{O}$, and pi-pi* stacking peaks at $284.9 \mathrm{eV}, 286.9 \mathrm{eV}$, and 289.6 $\mathrm{eV}$, respectively, confirm the successful thermal reduction of GO. The fitting of the high-resolution XPS O 1s spectrum was consistent with the $C 1$ s results.

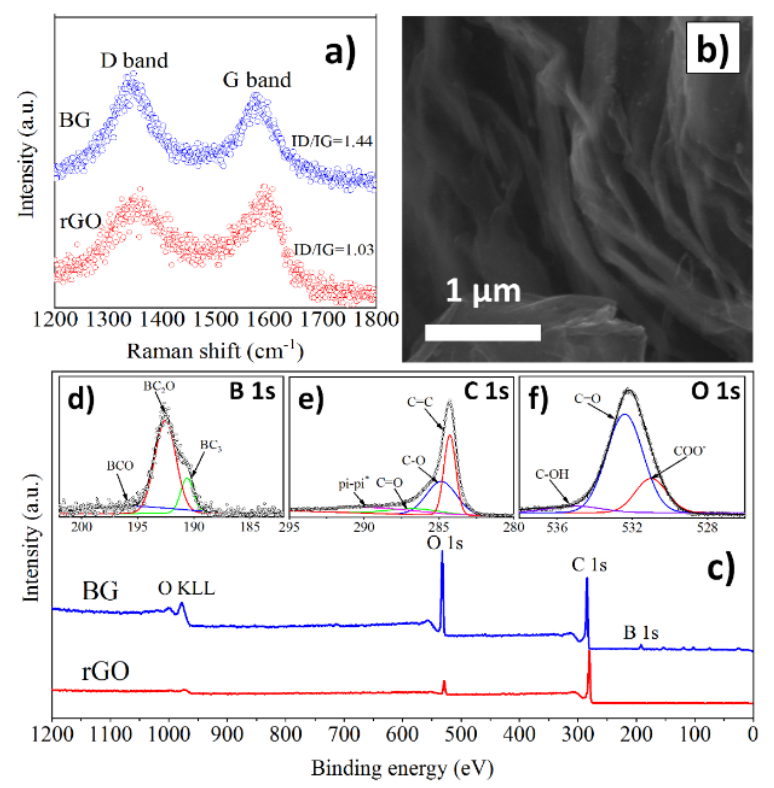

Fig. 4 (a) Raman spectra of $r G O$ and BG, (b) SEM image of BG, (c) XPS survey spectra of rGO and BG. Inset shows the high-resolution (d) B 1s, (e) C 1s, and (f) O 1s of BG.
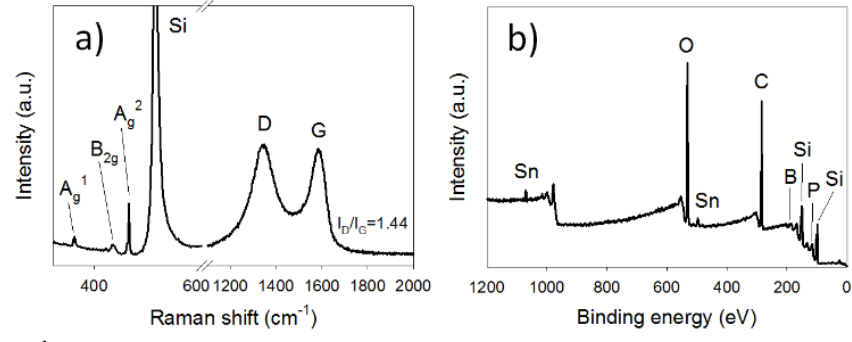

C)
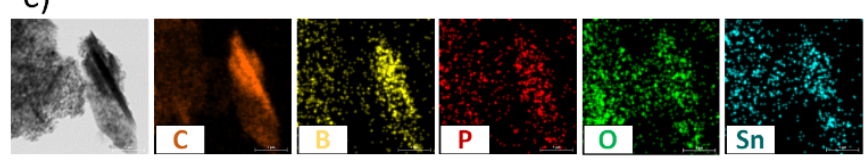

Fig. 5 (a) Raman spectrum, (b) XPS survey spectrum, and (c) EDX elemental mapping of FL-BP@BG sample.

The successful hybridization of FL-BP with BG was proven by Raman spectroscopy, XPS and EDX elemental mapping as shown in Fig. 5. As shown in Fig. 5a, all three Raman vibrational modes 
of FL-BP nanosheets and two characteristics bands of BG can be observed, suggesting that the FL-BP and BG were well incorporated. Moreover, it can be seen that the intensity of $\mathrm{Si}$ peak at around $520 \mathrm{~cm}^{-1}$ was relatively high despite FL-BP@BG was coated on the Si substrate. Fig. $5 b$ displays the XPS survey scan of the FL-BP@BG, revealing the existence of C, O, B, P and $\mathrm{Sn}$ elements. The appearance of $\mathrm{Si}$ peak is due to the $\mathrm{Si}$ substrate used for the sample preparation, while Sn can be attributed to the BP crystal. In BG, a doping concentration of boron was measured from the XPS analysis to be around 6\%, which is consistent with literature. ${ }^{41}$ As depicted in Fig. $5 c$, the elemental mapping on the FL-BP@BG sample also showed the existence of all peaks observed from the XPS survey scan, further confirming the successful hybridization of the BG and FLBP nanosheets.

We investigated the electrocatalytic activities of five different samples including FL-BP, rGO, BG, FL-BP@rGO and FL-BP@BG for HER in acidic media (in $0.5 \mathrm{M} \mathrm{H}_{2} \mathrm{SO}_{4}$ ). Fig. 6a shows the linear sweep voltammetry (LSV) curves of the catalysts loaded on a glassy carbon (GC) electrode. As a control electrocatalyst, the LSV curve of a traditional Pt/C was first recorded (Fig. S5). The electrocatalytic performance (overpotentials at $10 \mathrm{~mA} \mathrm{~cm}{ }^{-2}$, a value commonly used to evaluate the activity $)^{42}$ of our catalysts have been summarized in Fig. 6b. As compared to the GC (inactive for HER), FL-BP nanosheets showed better catalytic activity for the HER. However, the potential required to reach a current density of $10 \mathrm{~mA} \mathrm{~cm}{ }^{-2}$ was high $(618.3 \mathrm{mV})$, revealing the poor electrocatalytic activity of the bare FL-BP nanosheets, as expected. The overpotential value of the $\mathrm{rGO}$ at $10 \mathrm{~mA} \mathrm{~cm}-2$ was also high at $622.8 \mathrm{mV}$. Interestingly, much lower overpotential $(523.0 \mathrm{mV})$ was recorded for the BG to achieve 10 $\mathrm{mA} \mathrm{cm}{ }^{-2}$ in comparison to the non-doped rGO. This demonstrates that electron deficient and size compatible heteroatoms induced higher conductivity with better electrochemical activity for the BG. ${ }^{22}$ Promisingly, the heterostructured electrocatalyst based on FL-BP@rGO presents a much lower overpotential $(517.5 \mathrm{mV})$ than both individual FLBP $(618.3 \mathrm{mV})$ and $\mathrm{rGO}(622.8 \mathrm{mV})$, demonstrating the effectiveness of hybridizing 2D materials. Indeed, our FLBP@BG exhibited superior electrocatalytic activity with the lowest overpotential of $385.9 \mathrm{mV}$ to reach $10 \mathrm{~mA} \mathrm{~cm}{ }^{-2}$ among all electrocatalysts. These results clearly reveal that while heteroatom doping is an effective way to enhance the catalytic sites of rGO, hybridizing doped $\mathrm{rGO}$ ( $B G$ in our case) with solution processed FL-BP nanosheets can exhibit remarkable improvement in the HER activity.
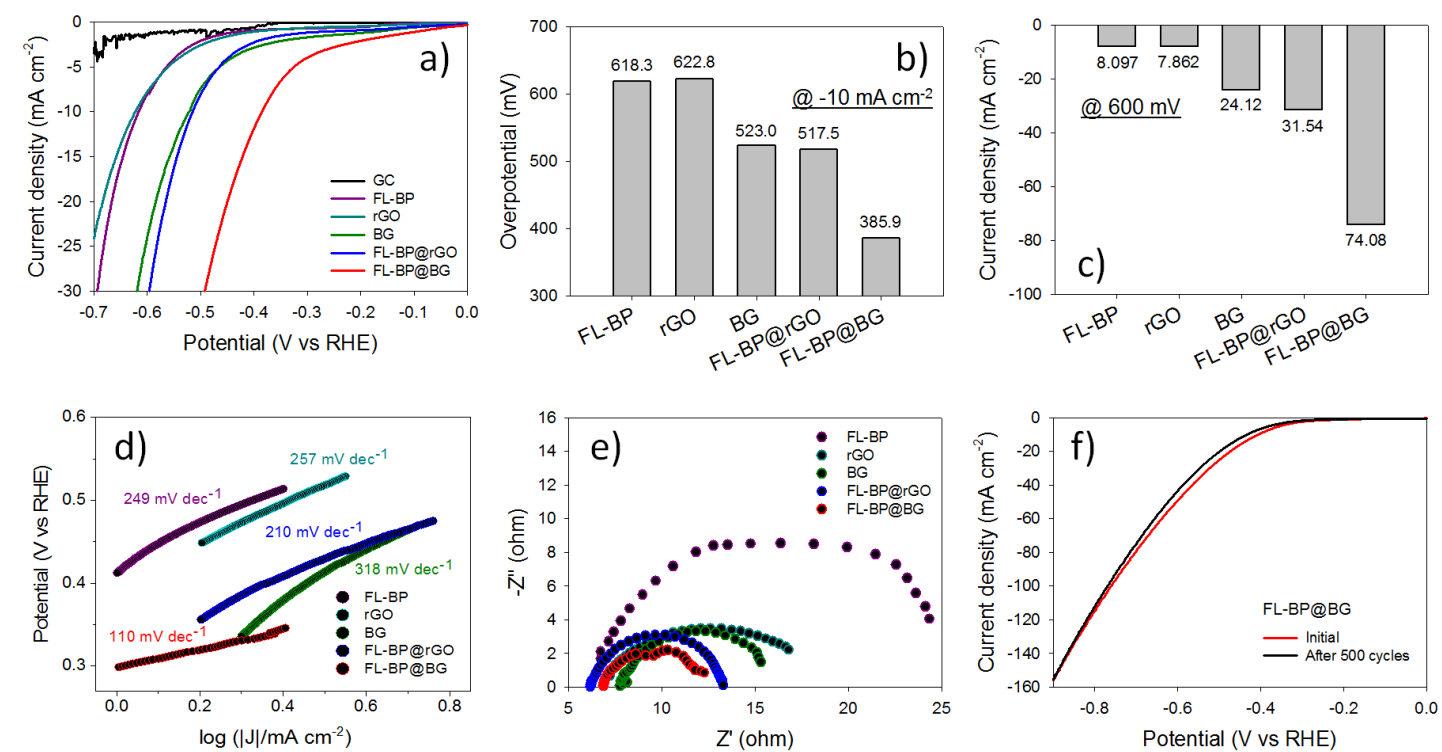

Fig. 6 (a) LSV curves, (b) overpotential values at a current density of $10 \mathrm{~mA} \mathrm{~cm}{ }^{-2}$, (c) current densities at a potential of $600 \mathrm{mV}$, (d) Tafel plots, and (e) EIS spectra of the five electrocatalysts including FL-BP, rGO, BG, FL-BP@rGO, and FL-BP@BG. (f) Polarization curves of of our FL-BP@BG catalyst initially and after 500 CV cycles.

The bar chart shown in Fig. 6c compares the current densities achieved by each catalyst at constant $600 \mathrm{mV}$. As depicted in Fig. $6 \mathrm{c}$, the bare FL-BP and rGO showed the lowest current density values (8.097 and $7.862 \mathrm{~mA} \mathrm{~cm}^{-2}$ ), respectively at 600 $\mathrm{mV}$, indicating their poor catalytic performance. Interestingly, the current densities of the heterostructured catalysts (FLBP@rGO and FL-BP@BG) were much higher than sum of the current densities of individual FL-BP and $\mathrm{rGO}$ or BG. For

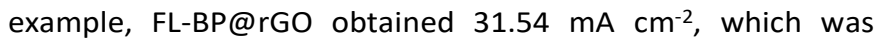
higher than the sum current density $\left(15.96 \mathrm{~mA} \mathrm{~cm}^{-2}\right)$ of individual FL-BP and $\mathrm{rGO}$. A current density of $74.08 \mathrm{~mA} \mathrm{~cm}^{-2}$ at $600 \mathrm{mV}$ was achieved using our FL-BP@BG heterocatalyst, while the FL-BP and BG showed 8.097 and $24.12 \mathrm{~mA} \mathrm{~cm}{ }^{-2}$, respectively. This suggests that the FL-BP nanosheets show excellent synergistic effect when integrated with another conductive material resulting in an excellent electrocatalytically active catalyst. ${ }^{43}$

Furthermore, the catalytic kinetics of our FL-BP, rGO, BG, FLBP@rGO and FL-BP@BG for HER were also investigated from the linear fitting of the Tafel plots (see Fig. 6d). The Tafel slope of the FL-BP@BG was measured to be $110 \mathrm{mV} \mathrm{dec}^{-1}$, which was significantly lower than that of the other catalysts tested in this work, revealing the outstanding activity of our heteroelectrocatalyst. In order to gain better insights into the 
intrinsic activity for the HER, electrochemical impedance spectroscopy (EIS) was conducted at constant voltage to measure the charge transfer resistance $\left(R_{c t}\right)$ of the catalysts in $0.5 \mathrm{M} \mathrm{H}_{2} \mathrm{SO}_{4}$ electrolyte. Promisingly, as illustrated in Fig. 6e, the FL-BP@BG exhibited the lowest $R_{\mathrm{ct}}$ value of $5.5 \Omega$ as compared to the other catalysts measured using the same experimental conditions, suggesting a favorable charge transfer mechanism for the FL-BP@BG system due to the effective hybridizing effect. On the other hand, the $R_{c t}$ of the bare FL-BP nanosheets was measured to be $18.0 \Omega$.

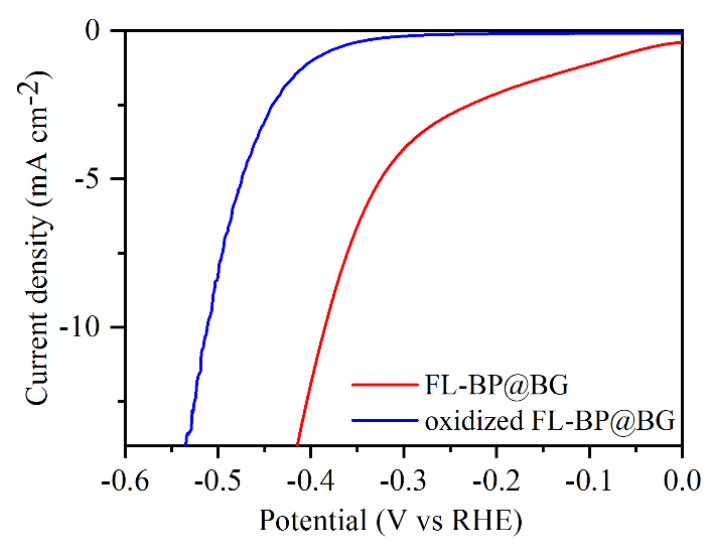

Fig. 7 LSV curves of non-oxidized (red) and oxidized (blue) FL-BP catalysts.

We further tested the stability of our FL-BP@BG catalyst by running 500 continuous cyclic voltammetry (CV) cycles in $0.5 \mathrm{M}$ $\mathrm{H}_{2} \mathrm{SO}_{4}$ electrolyte. Fig. $6 \mathrm{f}$ shows the HER polarization curves of our heteroelectrocatalyst before and after $500 \mathrm{CV}$ cycles. It can be seen from Fig. $6 f$ that no significant change in the electrocatalytic performance of the FL-BP@BG was observed after $500 \mathrm{CV}$ cycles, revealing that our heterocatalyst is very stable in acidic media during electrochemical reactions. The excellent stability of our FL-BP@BG can be explained by recent finding by a group of researchers who reported that the semiconducting BP nanosheets can be stable when it is in a hybrid form. ${ }^{44}$ For comparison, we also tested the electrocatalytic performance of oxidized FL-BP nanosheets incorporated BG. Recently, Xu et al. ${ }^{9}$ found using a DFT calculation that heteroatom doping including oxygen can increase the hydrophobicity of the phosphorene surface. Surface hydrophobicity has significant influence on the prefactor of kinetics of the reactants in electrochemistry. For instance, it was found by $\mathrm{Xu}$ et al. ${ }^{9}$ that surfaces with higher hydrophobicities (O-doped and $\mathrm{N}$-doped phosphorene) are helpful for the electrocatalytic NRR process, but the HER process is relatively hindered at these surfaces. As such, we carried out LSV measurement on the oxidized FL-BP@BG (see Fig. S2) coated GC electrode in $0.5 \mathrm{M} \mathrm{H}_{2} \mathrm{SO}_{4}$. It can be seen from Fig. 7 that an overpotential of $512 \mathrm{mV}$ was required to achieve $10 \mathrm{~mA} \mathrm{~cm}{ }^{-2}$ using the oxidized FL-BP nanosheets incorporated $B G$. This is much higher overpotential as compared to the nonoxidized FL-BP@BG (385.9 mV), confirming that the oxidation of FL-BP nanosheets is undesired for the HER process.

\section{Conclusions}

We have successfully prepared heteroatom (boron) doped graphene from naturally abundant graphite flakes (obtained from a Mongolian mine) using a combination of chemical and thermal treatments. Meanwhile, we have also used a facile exfoliation method to produce solution processed 2D FL-BP nanosheets with minimal oxidation. The electrocatalytic properties of the FL-BP nanosheets and their heterostructures with graphene derivatives ( $\mathrm{FGO}$ and $\mathrm{BG}$ ) for the HER in acidic media have been studied. Promisingly, the FL-BP@BG heteroelectrocatalyst catalyst exhibited an enhanced HER activity with a low overpotential of $385.9 \mathrm{mV}$ at $10 \mathrm{~mA} \mathrm{~cm}{ }^{-2}$, while showing an outstanding stability over 500 continuous CV cycles. This work demonstrates that hybridizing 2D FL-BP with heteroatom doped graphene is an efficient strategy to design stable BP based electrocatalyst. Importantly, this work also reveals that protecting FL-BP nanosheets from oxidation is of significant importance in achieving promising HER performance.

\section{Experimental}

\section{Preparation of FL-BP nanosheets}

FL-BP nanosheets were prepared using a liquid-phase exfoliation of bulk BP $(99.998 \%$, Smart Elements) in a solvent mixture of ethanol (99.7\%, XiLong Chemical Factory Co. Ltd.) and distilled (DI) water $(4: 1 \mathrm{v} / \mathrm{v})$. The solvent mixture was degassed using a continuous flow of argon (Ar) gas before being used. $50 \mathrm{mg}$ bulk BP crystal was ground and added into $10 \mathrm{~mL}$ of the mixture solvent. The exfoliation was carried out using a bath ultrasonicator (WUC-D03H, $425 \mathrm{~W}, 40 \mathrm{kHz}$ ) for $5 \mathrm{~h}$. After exfoliation, the solution was centrifuged (HC-3018R) at 6000 rpm for $30 \mathrm{~min}$. The stable dispersion of FL-BP nanosheets was obtained by collecting the supernatant of the centrifuged solution.

\section{Preparation of graphene oxide (GO)}

In this work, naturally abundant graphite flakes obtained from a mine located in Tuv aimag (Bayan soum), Mongolia were used. ${ }^{23}$ Specifically, the mine name: Zulegt, longitude: 470400 , latitude: 10739 05, above sea level: 1400-1460 m. The raw graphite was chemically oxidized to produce graphite oxide according to our recent work. ${ }^{23}$ In brief, a mixture of sulfuric acid (98\% $\mathrm{H}_{2} \mathrm{SO}_{4}$; XiLong Chemical Factory Co. Ltd) and phosphoric acid (85\%, $\mathrm{H}_{3} \mathrm{PO}_{4}$; UnionLab Chemical Factory Co. Ltd.) with a volume ratio $360: 40 \mathrm{~mL}$ (9:1) was poured into a beaker containing a mixture of raw graphite $(3 \mathrm{~g})$ and potassium permanganate $\left(99.5 \%, \mathrm{KMnO}_{4}, 18 \mathrm{~g}\right)$. The oxidation process was conducted under stirring the mixture at $330 \mathrm{rpm}$ at $50^{\circ} \mathrm{C}$ for 12 h. The reaction mixture was then cooled down to room temperature and poured onto ice $(300 \mathrm{~mL})$ with hydrogen peroxide $\left(30 \%, \mathrm{H}_{2} \mathrm{O}_{2}\right.$; XiLong Chemical Factory Co.Ltd, $3 \mathrm{~mL}$ ). Then the oxidized graphite was washed twice with the following solvents: (i) DI water, (ii) hydrochloric acid (30\%, $\mathrm{HCl}$; XiLong Chemical Factory Co. Ltd.) and (iii) ethyl alcohol. During washing steps, a white, insoluble, solid residue was observed at the bottom of the centrifuge tube and was removed from the sample. The final graphite oxide solution was bath sonicated to obtain graphene oxide (GO), followed by centrifuging at 4500 
rpm for $90 \mathrm{~min}$. The obtained light brown GO was freeze-dried (LGJ-12) at $-46{ }^{\circ} \mathrm{C}$ for $12 \mathrm{~h}$.

\section{Preparation of reduced graphene oxide (rGO), BG and hybrid materials}

The as-prepared $\mathrm{GO}$ was mixed adequately with boric acid (99.5\%, $\left.\mathrm{H}_{3} \mathrm{BO}_{3}\right)$ with a weight ratio of 1:10 using a mortar and pestle. Then the mixture ( $\mathrm{GO}$ and $\left.\mathrm{H}_{3} \mathrm{BO}_{3}\right)$ was put in an $\mathrm{Al}_{2} \mathrm{O}_{3}$ combustion boat and annealed in a tube furnace (PT-1200T, quartz tube) at $900{ }^{\circ} \mathrm{C}$ for $3 \mathrm{~h}$ under an $\mathrm{Ar}$ gas flow to obtain boron-doped graphene (BG). After cooling down to room temperature, the annealed sample was washed with hot water $\left(70{ }^{\circ} \mathrm{C}\right)$ to remove any residual $\mathrm{B}_{2} \mathrm{O}_{3}$. Reduced graphene oxide ( $\mathrm{rGO}$ ) was prepared using the same procedure (annealing GO at $900^{\circ} \mathrm{C}$ for 3 under $\mathrm{Ar}$ flow), but in the absence of boric acid. FLBP@rGO and FL-BP@BG were prepared by sonicating the mixture of previously prepared FL-BP nanosheets and $\mathrm{rGO}$ or BG for $1 \mathrm{~h}$. The mass ratio of the FL-BP and rGO or BG was 1:5.

\section{Materials characterization}

Raman spectra of the samples were obtained using a WITec alpha300 RA + S Raman microscope using an excitation laser wavelength of $532 \mathrm{~nm}$ with a $50 \times$ objective. The grating used was 600 grooves $\mathrm{mm}^{-1}$. The sample for Raman spectroscopy was prepared on silicon ( $\mathrm{Si}$ ) substrates by drop casting.

X-ray photoelectron spectroscopy (XPS) was carried out using a Kratos Axis ULTRA X-ray Photoelectron Spectrometer incorporating a $165 \mathrm{~mm}$ hemispherical electron energy analyzer. The incident radiation was monochromatic Al $\mathrm{K}_{\alpha} \mathrm{X}$ rays $(1486.6 \mathrm{eV})$ at $225 \mathrm{~W}(15 \mathrm{kV}, 15 \mathrm{~mA})$. The base pressure in the analysis chamber was $1.0 \times 10^{-8}$ Torr. Survey scans were collected at an analyzer pass energy of $160 \mathrm{eV}$ while highresolution (HR) scans were at $20 \mathrm{eV}$. Survey scans were carried out at binding energies between $1200 \mathrm{eV}$ and $0 \mathrm{eV}$ with $1.0 \mathrm{eV}$ steps and $100 \mathrm{~ms}$ dwell time. HR scans were run with $0.05 \mathrm{eV}$ steps and $250 \mathrm{~ms}$ dwell time.

Bright-field transmission electron microscopy (TEM) images were acquired using a FEI Titan Themis. Scanning transmission electron microscopy (STEM) imaging and Energy-Dispersive $X$ ray (EDX) elemental mapping were carried out also on a $\mathrm{FEI}$ Titan Themis S-TEM instrument. The STEM probe was aberration corrected, enabling sub-angstrom spatial resolution, and HAADF images were obtained.

\section{Electrochemical measurement}

The electrochemical tests were performed in a three-electrode configuration connected to an electrochemical workstation ( $\mathrm{CHI}$ 760D). $\mathrm{Ag} / \mathrm{AgCl}$ electrode, carbon paper and catalyst coated glassy carbon (GC) were used as the reference, counter and working electrodes, respectively. The potentials recorded against an $\mathrm{Ag} / \mathrm{AgCl}$ electrode were converted to the reversible hydrogen electrode (RHE) using the Nernst equation: $\mathrm{E}_{\mathrm{RHE}}=$ $\mathrm{E}_{\mathrm{Ag} / \mathrm{AgCl}}+0.0591 \mathrm{pH}+0.196 \mathrm{~V}$. All data were collected with iRcompensation. The working electrodes were prepared as follows: $5 \mathrm{mg}$ of catalyst and $70 \mu \mathrm{L}$ Nafion solution ( $5 \mathrm{wt} \%$ ) were dispersed in $1 \mathrm{~mL}$ of water/isopropanol mixture solution (volume ratio of $4: 1$ ) under $10 \mathrm{~min}$ bath sonication to produce a catalyst ink. Then, $12 \mu \mathrm{L}$ of catalyst ink was loaded onto a GC electrode with an area of $0.196 \mathrm{~cm}^{2}$. The catalyst loading was $0.285 \mathrm{mg} \mathrm{cm}^{-2}$. Linear sweep voltammetry (LSV) with a scan rate of $5 \mathrm{mV} \mathrm{s}^{-1}$ was carried out using a rotation disk electrode (RDE) at a rotation speed of $1600 \mathrm{rpm}$ in $0.5 \mathrm{M} \mathrm{H}_{2} \mathrm{SO}_{4}$. Electrochemical impedance spectroscopy (EIS) was performed in the frequency range from $100 \mathrm{kHz}$ to $0.01 \mathrm{~Hz}$.

\section{Conflicts of interest}

There are no conflicts to declare.

\section{Acknowledgements}

This research was partially supported by Fellow research grant of National University of Mongolia (P2019-3648). The support of the Australian Research Council Discovery Program (DP160101301) is gratefully acknowledged. The authors thank Dr. Ashley Slattery of Adelaide Microscopy at the University of Adelaide for his help with TEM analysis. We gratefully acknowledge the use of the Queensland node of the Australian National Fabrication Facility (ANFF-Q) and Centre for Microscopy and Microanalysis (CMM) at the University of Queensland, Australia.

\section{Notes and references}

1. X. Zou and Y. Zhang, Chem. Soc. Rev., 2015, 44, 5148-5180.

2. J. R. McKone, E. L. Warren, M. J. Bierman, S. W. Boettcher, B. S. Brunschwig, N. S. Lewis and H. B. Gray, Energy Environ. Sci., 2011, 4, 3573-3583.

3. J. Zhang, Y. Zhao, X. Guo, C. Chen, C.-L. Dong, R.-S. Liu, C.P. Han, Y. Li, Y. Gogotsi and G. Wang, Nat Catal., 2018, 1, 985-992.

4. Y. Jiao, Y. Zheng, K. Davey and S.-Z. Qiao, Nat Energy, 2016, 1, 16130.

5. Y. Chen, K. Yang, B. Jiang, J. Li, M. Zeng and L. Fu, J. Mater. Chem. A., 2017, 5, 8187-8208.

6. H. Shuai, J. Li, F. Jiang, X. Zhang, L. Xu, J. Hu, H. Hou, G. Zou, W. Sun, H. Duan, J. Hu and X. Ji, Sci. China Mater., 2020, DOI: 10.1007/s40843-020-1347-5.

7. H. Shuai, P. Ge, W. Hong, S. Li, J. Hu, H. Hou, G. Zou and X. Ji, Small Methods, 2019, 3, 1800328.

8. Z. Huang, H. Hou, Y. Zhang, C. Wang, X. Qiu and X. Ji, Adv. Mater., 2017, 29, 1702372.

9. G. Xu, H. Li, A. S. R. Bati, M. Bat-Erdene, M. J. Nine, D. Losic, Y. Chen, J. G. Shapter, M. Batmunkh and T. Ma, J. Mater. Chem. A., 2020, 8, 15875-15883.

10. K. Zhang, B. Jin, C. Park, Y. Cho, X. Song, X. Shi, S. Zhang, W. Kim, H. Zeng and J. H. Park, Nat Commun., 2019, 10, 2001.

11. Y. Peng, B. Lu, N. Wang, J. E. Lu, C. Li, Y. Ping and S. Chen, ACS Appl. Mater. Interfaces., 2019, 11, 24707-24714.

12. R. He, J. Hua, A. Zhang, C. Wang, J. Peng, W. Chen and J. Zeng, Nano Lett., 2017, 17, 4311-4316.

13. X. Zhu, T. Zhang, Z. Sun, H. Chen, J. Guan, X. Chen, H. Ji, P. Du and S. Yang, Adv. Mater., 2017, 29, 1605776.

14. M. Batmunkh, M. Bat-Erdene and J. G. Shapter, Adv. Energy Mater., 2018, 8, 1701832.

15. C. C. Mayorga-Martinez, N. Mohamad Latiff, A. Y. S. Eng, Z. Sofer and M. Pumera, Anal. Chem., 2016, 88, 10074-10079. 
Y. Cai, J. Gao, S. Chen, Q. Ke, G. Zhang and Y.-W. Zhang, Chem. Mater., 2019, 31, 8948-8956.

17. J. Lu, X. Zhang, D. Liu, N. Yang, H. Huang, S. Jin, J. Wang, P. K. Chu and X.-F. Yu, ACS Appl. Mater. Interfaces., 2019, 11, 37787-37795.

18. L. Shao, H. Sun, L. Miao, X. Chen, M. Han, J. Sun, S. Liu, L. Li, F. Cheng and J. Chen, J. Mater. Chem. A., 2018, 6, 24942499.

19. X. Wang, L. Bai, J. Lu, X. Zhang, D. Liu, H. Yang, J. Wang, P. K. Chu, S. Ramakrishna and X.-F. Yu, Angew. Chem. Int. Ed., 2019, 58, 19060-19066.

20. Y. Li, W. Pei, J. He, K. Liu, W. Qi, X. Gao, S. Zhou, H. Xie, K. Yin, Y. Gao, J. He, J. Zhao, J. Hu, T.-S. Chan, Z. Li, G. Zhang and M. Liu, ACS Catal., 2019, 9, 10870-10875.

21. X.-D. Zhu, Y. Xie and Y.-T. Liu, J. Mater. Chem. A., 2018, 6, 21255-21260.

22. Z. Yuan, J. Li, M. Yang, Z. Fang, J. Jian, D. Yu, X. Chen and L. Dai, J. Am. Chem. Soc., 2019, 141, 4972-4979.

23. S. Suragtkhuu, O. Tserendavag, U. Vandandoo, A. S. R. Bati, M. Bat-Erdene, J. G. Shapter, M. Batmunkh and S. Davaasambuu, RSC Adv., 2020, 10, 9133-9139.

$24 . \quad J$. D. Wood, S. A. Wells, D. Jariwala, K.-S. Chen, E. Cho, V. K. Sangwan, X. Liu, L. J. Lauhon, T. J. Marks and M. C. Hersam, Nano Lett., 2014, 14, 6964-6970.

25. M. Batmunkh, M. Bat-Erdene and J. G. Shapter, Adv. Mater., 2016, 28, 8586-8617.

26. H. Liu, A. T. Neal, Z. Zhu, Z. Luo, X. Xu, D. Tománek and P. D. Ye, ACS Nano, 2014, 8, 4033-4041.

27. M. Batmunkh, A. Shrestha, M. Bat-Erdene, M. J. Nine, C. J. Shearer, C. T. Gibson, A. D. Slattery, S. A. Tawfik, M. J. Ford, S. Dai, S. Qiao and J. G. Shapter, Angew. Chem. Int. Ed., 2018, 57, 2644-2647.

28. Z. Sofer, J. Luxa, D. Bouša, D. Sedmidubský, P. Lazar, T. Hartman, H. Hardtdegen and M. Pumera, Angew. Chem. Int. Ed., 2017, 56, 9891-9896.

29. Z. Guo, H. Zhang, S. Lu, Z. Wang, S. Tang, J. Shao, Z. Sun, H. Xie, H. Wang, X.-F. Yu and P. K. Chu, Adv. Funct. Mater., 2015, 25, 6996-7002.

30. M. Batmunkh, K. Vimalanathan, C. Wu, A. S. R. Bati, L. Yu, S. A. Tawfik, M. J. Ford, T. J. Macdonald, C. L. Raston, S. Priya, C. T. Gibson and J. G. Shapter, Small Methods, 2019, 3, 1800521

31. H. Huang, Q. Xiao, J. Wang, X.-F. Yu, H. Wang, H. Zhang and P. K. Chu, npj 2D Mater Appl., 2017, 1, 20.

32. M. Bat-Erdene, M. Batmunkh, C. J. Shearer, S. A. Tawfik, M. J. Ford, L. Yu, A. J. Sibley, A. D. Slattery, J. S. Quinton, C. T. Gibson and J. G. Shapter, Small Methods, 2017, 1, 1700260.

33. Z. H. Sheng, H. L. Gao, W. J. Bao, F. B. Wang and X. H. Xia, Journal of Materials Chemistry, 2012, 2, 390-395.

34. G. Katagiri, H. Ishida and A. Ishitani, Carbon, 1988, 26, 565571.

35. A. C. Ferrari, J. C. Meyer, V. Scardaci, C. Casiraghi, M. Lazzeri, F. Mauri, S. Piscanec, D. Jiang, K. S. Novoselov, S. Roth and A. K. Geim, Phys. Rev. Lett., 2006, 97, 187401.

36. A. Das, S. Pisana, B. Chakraborty, S. Piscanec, S. K. Saha, U. V. Waghmare, K. S. Novoselov, H. R. Krishnamurthy, A. K. Geim, A. C. Ferrari and A. K. Sood, Nature Nanotech., 2008, 3, 210-215

37. Y. Hishiyama, H. Irumano, Y. Kaburagi and Y. Soneda, Phys. Rev. B., 2001, 63, 245406.

38. M. Cattelan, S. Agnoli, M. Favaro, D. Garoli, F. Romanato, M. Meneghetti, A. Barinov, P. Dudin and G. Granozzi, Chem. Mater., 2013, 25, 1490-1495.
39. L. Zhang, Z.-Y. Zhang, R.-P. Liang, Y.-H. Li and J.-D. Qiu, Anal. Chem., 2014, 86, 4423-4430.

S. Agnoli and M. Favaro, J. Mater. Chem. A., 2016, 4, 50025025.

41. X. Yu, P. Han, Z. Wei, L. Huang, Z. Gu, S. Peng, J. Ma and G. Zheng, Joule, 2018, 2, 1610-1622.

42. J. Yan, L. Kong, Y. Ji, Y. Li, J. White, S. Liu, X. Han, S.-T. Lee and T. Ma, Commun. Chem., 2018, 1, 95.

43. M. Batmunkh, M. Myekhlai, A. S. R. Bati, S. Sahlos, A. D. Slattery, Tania M. Benedetti, V. R. Gonçales, C. T. Gibson, J. J. Gooding, R. D. Tilley and J. G. Shapter, J. Mater. Chem. A., 2019, 7, 12974-12978.

44. H. Uk Lee, S. C. Lee, J. Won, B.-C. Son, S. Choi, Y. Kim, S. Y. Park, H.-S. Kim, Y.-C. Lee and J. Lee, Sci Rep., 2015, 5, 8691. 